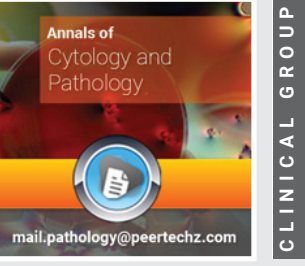

\title{
Development of a management guide wheel for cervical cancer
}

\section{Mahmoud Samy Ismail ${ }^{1-4 \star}$, Fouad Ismail ${ }^{5}$, Julia Ismail ${ }^{6}$, Muneera AlKhalifa ${ }^{7}$, Rehab Ismael ${ }^{1}$, Reham Fathi ${ }^{1}$, Wassan Al Ani' ${ }^{1}$ Shaikha Al Hajri', Nusiba Elhassan ${ }^{1}$, Mooza AlKawari ${ }^{1}$, Aysha AlBinali ${ }^{1}$, Gulmeen Raza ${ }^{1}$, Mariam Fida ${ }^{2}$, Alaa Zeineldine ${ }^{1}$ and Uwe Torsten ${ }^{3,4,8}$ \\ 'Department of Obstetrics and Gynecology, King Hamad University Hospital (KHUH), Kingdom of Bahrain}

${ }^{2}$ Bahrain Oncology Centre (BOC), Kingdom of Bahrain

${ }^{3}$ Department of Gyne-Oncology, Charitè University Hospital, Humboldt University, Berlin, Germany ${ }^{4}$ Pan Arabian Research Society of Gynecological Oncology (PARSGO), Kingdom of Bahrain and Berlin, Germany

${ }^{5}$ University of Applied Arts, Vienna, Austria

${ }^{6}$ Vienna University of Technology, Vienna, Austria

${ }^{7}$ King Hamad University Hospital (KHUH), Kingdom of Bahrain

${ }^{8} \mathrm{Head}$ of Department of Obstetrics and Gynecology, Royal College of Surgeons in Ireland (RCSI),

Kingdom of Bahrain
Received: 21 December, 2021

Accepted: 08 February, 2022

Published: 09 February, 2022

*Corresponding author: Mahmoud Samy Ismail, Department of Obstetrics and Gynecology, King Hamad University Hospital (KHUH),

Kingdom of Bahrain, Tel: +97335593048 ;

E:mail: samyismael@hotmail.com

Copyright License: (c) 2022 Ismail MS, et al. This is an open-access article distributed under the terms of the Creative Commons Attribution License, which permits unrestricted use, distribution, and reproduction in any medium, provided the original author and source are credited.

Keywords: Cervical cancer; Surgical staging; Radiotherapy; Chemotherapy; Systemic therapy; FIGO classification; Guidelines; Persistent or recurrent disease; Therapy for relapse; Surveillance; Surgical node status; Trachelectomy; Radical hysterectomy; Distant metastases

https://www.peertechzpublications.com

Check for updates

\section{Abstract}

We designed and developed a management guide wheel for cervical cancer to facilitate a standard approach and improve the quality of care in managing patients diagnosed with different stages of cervical cancer. Each step of the wheel considers the patient's current medical condition, FIGO stage, and the possible treatment modalities for cervical cancer in her case. We reviewed existing international guidelines on the management of cervical cancer and compared their respective recommendations. This guide wheel is based on recommendations by the following organizations: the National Comprehensive Cancer Network (NCCN), the Society of Gynecologic Oncology (SGO), the European Society of Gynaecological Oncology (ESGO), the European Society for Medical Oncology (ESMO), the American Society of Clinical Oncology (ASCO), the British Gynaecological Cancer Society (BGCS) and the International Federation of Gynecology and Obstetrics (FIGO).

\section{Abbreviations}

ASCO: American Society of Clinical Oncology; BGCS: British Gynaecological Cancer Society; CC: cervical cancer; CT: Computed Tomography; EBRT: External Beam Radiotherapy; ESGO: European Society of Gynaecological Oncology; ESMO: European Society for Medical Oncology; FIGO: International Federation of Gynecology and Obstetrics; IORT: IntraOperative Radiotherapy; LN: Lymph Node; LND: Lymph Node Dissection; LVSI: Lymphovascular Space Invasion; MRI: Magnetic Resonance Imaging; NCCN: National Comprehensive Cancer Network; PET: Positron Emission Tomography; RH: Radical Hysterectomy; SGO: Society of Gynecologic Oncology; TNM: Tumors, Nodes, and Metastases.

\section{Introduction}

Cervical Cancer (CC) is the fourth most common cancer in women worldwide and the second most common cancer in developing countries [1-7]. The mainstay of treating CC involves surgery, radiotherapy, chemotherapy, or a combination of those [6-9]. Early-stage CC is usually asymptomatic and patients present with symptoms in advanced stages $[4,5,10]$. CC can be preventable with effective screening and vaccination programs $[4,5,11,12]$. Early detection of pre-malignant states before they develop into cancer leads to earlier treatment and hence, a better outcome [12]. In fact, early-stage CC has a cure rate of $80-95 \%$ [8]. $30 \%$ of CC in developed countries are Stage IB or higher compared to $60 \%$ in developing countries due to 
the unavailability of well-established screening programs in the latter $[2,12]$.

Various health organizations globally have developed sets of guidelines on the management of CC. We compared recommendations by the National Comprehensive Cancer Network (NCCN), the Society of Gynecology (SGO), the European Society of Gynaecological Oncology (ESGO), the European Society for Medical Oncology (ESMO), the American Society of Clinical Oncology (ASCO), the British Gynaecological Cancer Society (BGCS) and the International Federation of Gynecology and Obstetrics (FIGO) $[4-6,9,10,13,14]$. The NCCN guidelines for managing cancer cervix is one of the most frequently updated and comprehensive guidelines available [4].

We developed a management guide wheel for CC based on the recommendations of the compared guidelines but mainly reflecting the most recently updated guidelines by NCCN. This guide wheel aims to serve healthcare professionals in finding a quick and comprehensive display of the recommended steps and alternatives to manage patients diagnosed with CC when counseling patients. We are also comparing the guidelines covered here in another research article to highlight the similarities and differences in their respective recommendations. We developed a similar guide wheel for the management of uterine neoplasms [15]. We also developed a guide wheel describing the management of abnormal pap smear results and a comparison of the recommendations of international organizations $[12,16]$.

\section{About this guide wheel}

This guide wheel includes recommendations and algorithms for managing patients with $\mathrm{CC}$ as per the guidelines compared during this study (Figure 1). It consists of a base (Figure 2) and an inner wheel (Figure 3). There is more information on how to use the guide wheel on the back of the base (Figure 4).

There are thirteen possible modalities of treatment with the different options under those modalities mentioned on the inner wheel as follows:

- Primary Therapy: a. Surgery; b. Radiotherapy; c. Chemotherapy; d. Imaging; e. Lymph node (LN) status

- Surgical Therapy: a. Conization; b. Re-Conization; c. Hysterectomy (A. Simple; B. Modified Radical Hysterectomy (RH); C. RH); d. Lymphadenectomy (Pelvic; Para-aortic); e. Trachelectomy; f. Completion; g. Resection

- Additional Workup: a. Imaging; b. Biopsy; c. Pathology; d. Surgical Staging; e. Exploration

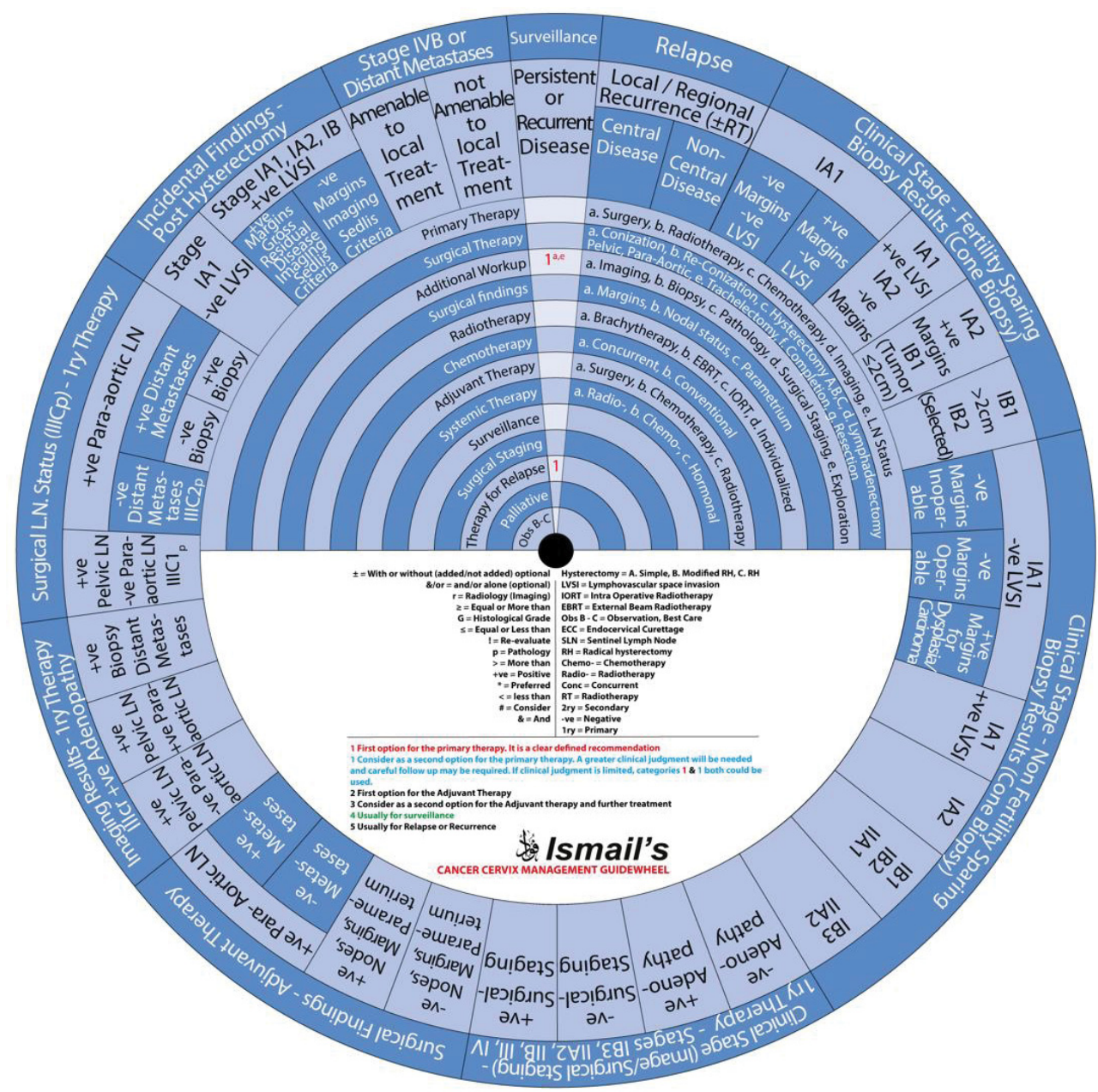

Figure 1: The guide wheel fully assembled demonstrates the surveillance pathway when managing persistent or recurrent cervical cancer.

Citation: Ismail MS, Ismail F, Ismail J, AlKhalifa M, Ismael R, et al. (2022) Development of a management guide wheel for cervical cancer. Ann Cytol Pathol 7(1): 014-028. DOI: https://dx.doi.org/10.17352/acp.000025 


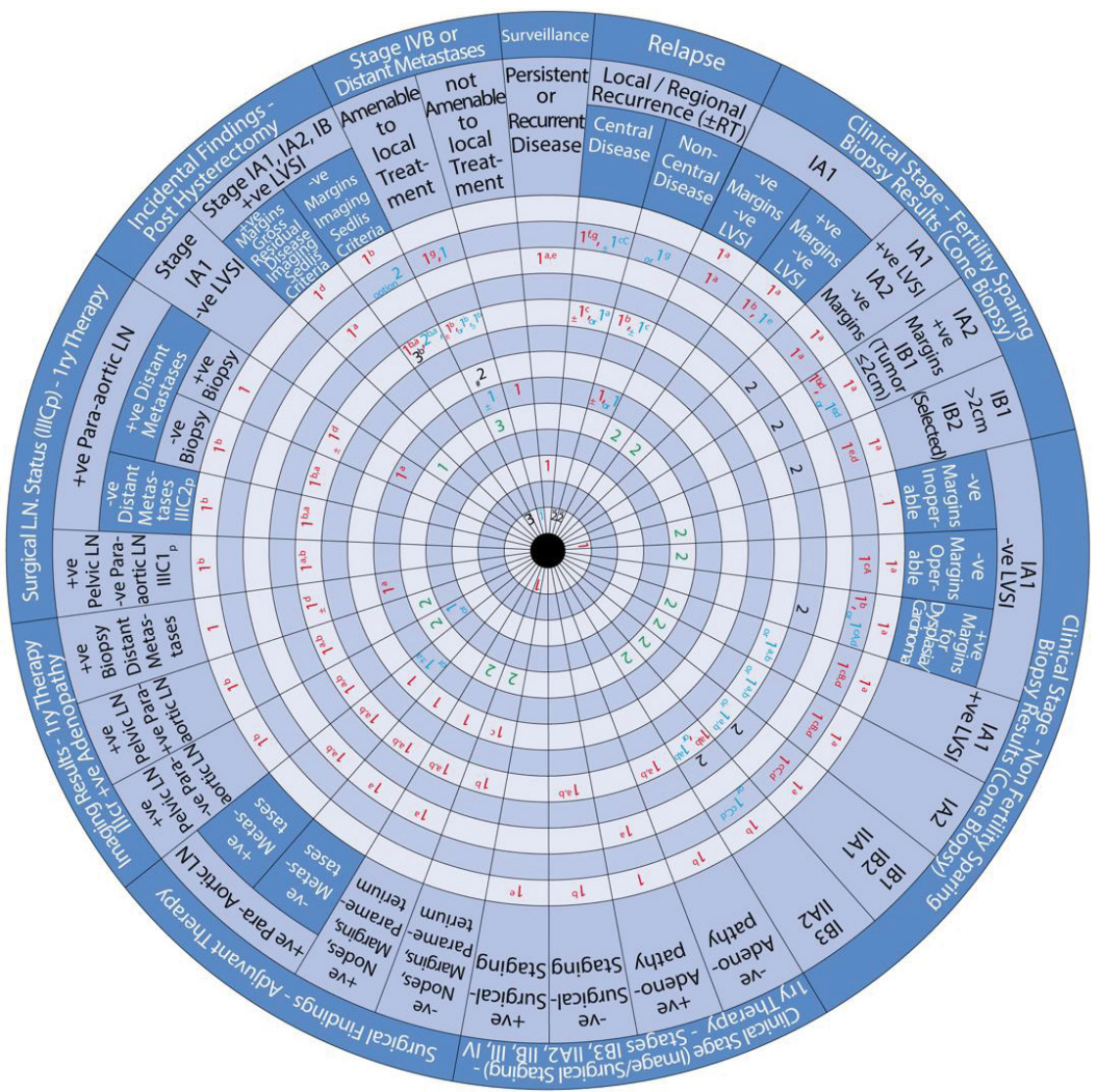

Figure 2: The base of the guide wheel demonstrates the stages of cervical cancer and the color-coded treatment modalities.

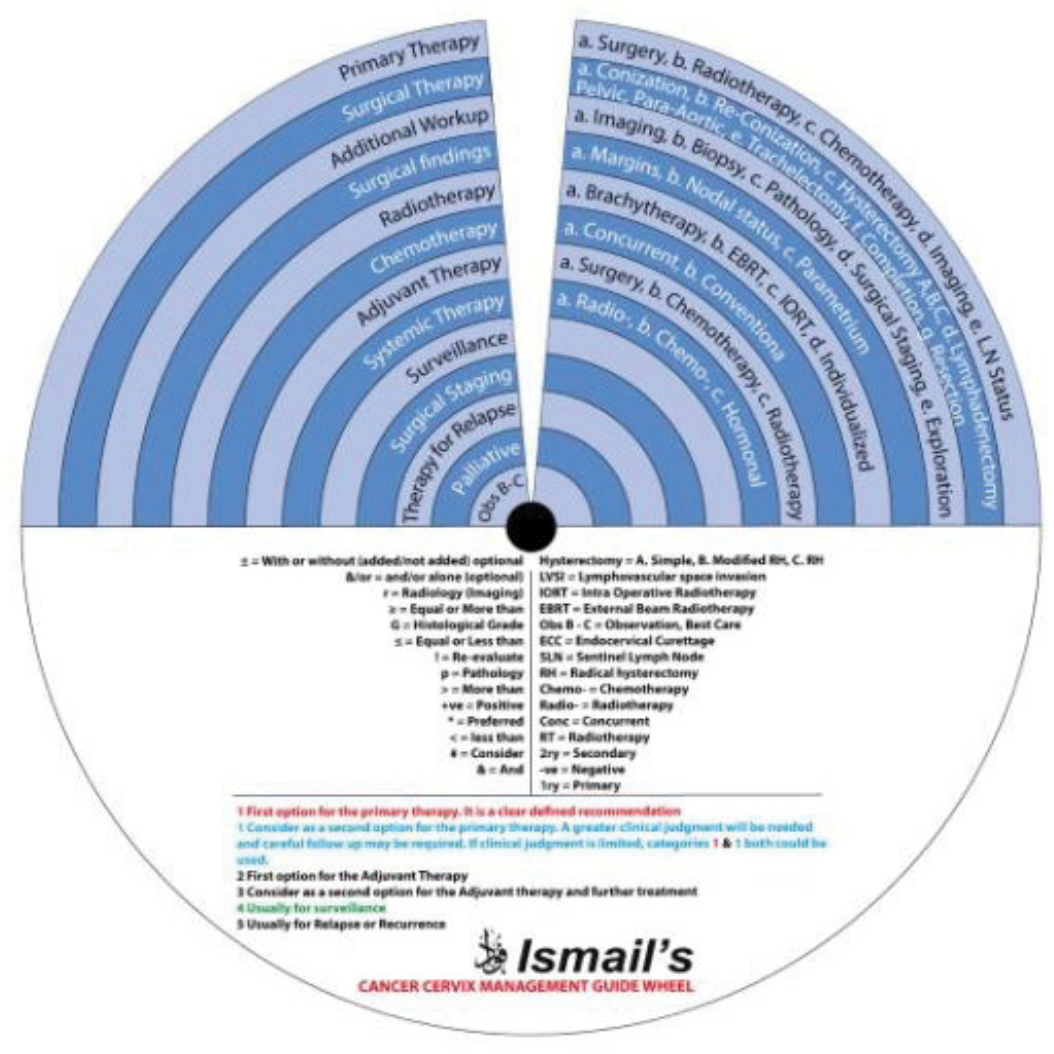

Figure 3: The inner wheel of the guide wheel that is placed on top of the base demonstrates the various treatment modalities and the available options for each.

Citation: Ismail MS, Ismail F, Ismail J, AlKhalifa M, Ismael R, et al. (2022) Development of a management guide wheel for cervical cancer. Ann Cytol Pathol 7(1): 014-028. DOI: https://dx.doi.org/10.17352/acp.000025 
Figure 4: The back of the guide wheel describes how to use the wheel in addition to the FIGO staging system of cervical cancer.

- Surgical findings: a. Margins; b. Nodal status; c. Parametrium

- Radiotherapy: a. Brachytherapy; b. External Beam Radiotherapy (EBRT); c. Intraoperative Radiotherapy (IORT); d. Individualized

- Chemotherapy: a. Concurrent; b. Conventional

- Adjuvant Therapy: a. Surgery; b. Chemotherapy; c. Radiotherapy

- Systemic Therapy: a. Radiotherapy; b. Chemotherapy; c. Hormonal

- Surveillance

- $\quad$ Surgical Staging

- Therapy for Relapse

- Palliative Therapy

- Observation, Follow-up and Supportive care.

\section{How to use this guide wheel}

The guide wheel aligns the treatment modalities on the inner wheel with the particular stage of cervical cancer diagnosed, on the outer wheel, following clinical, radiological, and histopathological investigations. The viewing slot displays the steps recommended to follow in chronological order to manage the patient under consideration (Figure 1).
The base of the guide wheel displays color-coded treatment modalities in order. The numbers signify the steps and alternative options as follows:

$1 \rightarrow$ This marks the first option for Primary Therapy. It is a clearly defined recommendation.

$1 \rightarrow$ This is a second option for Primary Therapy. It might require greater clinical judgment and careful follow-up. If the clinical judgment is limited, either option 1 or 1 could be used.

$2 \rightarrow$ This marks the first option for Adjuvant Therapy.

$3 \rightarrow$ This is considered as a second option for Adjuvant Therapy and further treatment.

$4 \rightarrow$ This is usually reserved for surveillance.

$5 \rightarrow$ This is usually reserved for Relapse or Recurrence.

Abbreviations used on the wheel include the following:

\begin{tabular}{|l|l|l|l|}
\hline \pm & $\begin{array}{l}\text { There is an option to } \\
\text { add or to not add (with } \\
\text { or without) }\end{array}$ & ! & Re-evaluate \\
\hline$>$ & More than & LVSI & Lymphovascular space invasion \\
\hline$<$ & Less than & ECC & Endocervical Curettage \\
\hline$\geq$ & Equal or more than & RH & Radical hysterectomy \\
\hline$\leq$ & Equal or less than & RT & Radiotherapy \\
\hline$\#$ & Consider & SLN & Sentinel Lymph Node \\
\hline
\end{tabular}




\begin{tabular}{|l|l|l|l|}
\hline E & Preferred & EBRT & External Beam Radiotherapy \\
\hline \& & And & IORT & Intra Operative Radiotherapy \\
\hline \&/or & And/or alone (optional) & 1 ry & Primary \\
\hline r & Radiology (Imaging) & 2 ry & Secondary \\
\hline p & Pathology & Conc & Concurrent \\
\hline G & Histological Grade & Radio- & Radiotherapy \\
\hline +ve & Positive & Chemo- & Chemotherapy \\
\hline -ve & Negative & Obs, B-C & Observation, Best Care \\
\hline
\end{tabular}

The stages of cervical cancer shown on the outer wheel are as follows:

1. Fertility-Sparing Options for Stages IA1, IA2, IB1, and select IB2 Based on Cone Biopsy Results

2. Non-Fertility Sparing Options for Stages IA1, IA2, IB1, IB2, IB3, IIA1, and IIA2 Based on Cone Biopsy Results

3. Primary Therapy for Stages IB3, IIA2, IIB, III, and IV Based on Imaging or Surgical Staging

4. Adjuvant Therapy Based on Surgical Findings

5. Primary Therapy Based on Imaging Results Demonstrating Positive Adenopathy

6. Primary Therapy Based on Surgical Lymph Node Status

7. Incidental Surgical Finding of Cervical Cancer PostHysterectomy

8. Stage IVB or Distant Metastases

9. Surveillance of Persistent or Recurrent Disease

\section{Therapy for Relapse}

The back of the wheel displays the FIGO staging system for cervical cancer, most recently updated in 2018, as followed by the international guidelines. The table below exhibits the stages of cervical cancer and their definitions $[1,4,6,7,9,10]$.

FIGO Stages and Sub-Classifications

Stage I: The carcinoma is strictly confined to the cervix uteri (extension to the corpus should be disregarded)

IA: Invasive carcinoma that can be diagnosed only by microscopy with a maximum depth of invasion $\leq 5 \mathrm{~mm}^{3}$

IA1: Measured stromal invasion $<3 \mathrm{~mm}$ in depth

IA2: Measured stromal invasion $\geq 3 \mathrm{~mm}$ and $<5 \mathrm{~mm}$ in depth

IB1: Invasive carcinoma $\geq 5 \mathrm{~mm}$ depth of stromal invasion and $<2 \mathrm{~cm}$ in greatest dimension

IB: Invasive carcinoma with measured deepest invasion $\geq 5 \mathrm{~mm}$ (greater than stage IA); lesion limited to the cervix uteri

IB2: Invasive carcinoma $\geq 2 \mathrm{~cm}$ and $<4$ $\mathrm{cm}$ in greatest dimension

IB3: Invasive carcinoma $\geq 4 \mathrm{~cm}$ in greatest dimension

Stage II: The cervical carcinoma invades beyond the uterus, but has not extended onto the lower third of the vagina or to the pelvic wall

IIA1: Invasive carcinoma $<4 \mathrm{~cm}$ in

IIA: Involvement limited to the upper two-thirds of the vagina without greatest dimension

IIA2: Invasive carcinoma $\geq 4 \mathrm{~cm}$ in greatest dimension
Stage III: The carcinoma involves the lower third of the vagina and/or extends to the pelvic wall and/or causes hydronephrosis or non-functioning kidney and/or involves pelvic and/or para-aortic lymph nodes

IIIA: Carcinoma involves the lower third of the vagina, with no extension to the pelvic wall

IIIC: Involvement of pelvic and/or para-aortic lymph nodes, irrespective

of tumor size and extent (with imaging IIIC2: Para-aortic lymph node metastasis and pathology notations)

Stage IV: The carcinoma has extended beyond the true pelvis or has involved (biopsy-proven) the mucosa of the bladder or rectum. Bullous edema, as such, does not permit a case to be allotted to stage IV

IVA: Spread of the growth to adjacent organs

IIIB: Extension to the pelvic wall and/ or hydronephrosis or non-functioning kidney (unless known to be due to another cause)

IIIC1: Pelvic lymph node metastasis only

Recommendations on managing patients with cervical cancer using the guide wheel

Sector 1: Fertility-Sparing Options for Stages IA1, IA2, IB1, and select IB2 Based on Cone Biopsy Results (Figure 5)

For patients with stage IA1 cervical cancer with negative margins and negative LVSI:

- 1a: Primary treatment is surgery (1a).

- 1a: Surgical treatment (1) with conization (a).

- 2: The second step is surveillance.

For patients with stage IA1 cervical cancer with positive margins and negative LVSI:

- 1a: Primary treatment is surgery (1a).

- 1b: Surgical treatment (1) with re-conization (b).

- 1e: An alternative is a surgical treatment (1) with trachelectomy (e).

- 2: The second step is surveillance.

For patients with stage IA1 cervical cancer with positive LVSI and stage IA2 with negative margins:

- 1a: Primary treatment is surgery (1a).

- 1e, d: Surgical treatment (1) with conization (e) with pelvic lymphadenectomy with or without para-aortic lymphadenectomy (d). Sentinel LN mapping can be considered.

- or 1a, d: An alternative is a surgical treatment (1) with conization (a) with pelvic lymphadenectomy. Sentinel LN mapping can also be considered if negative margins.

- 2: The second step depends on surgical findings.

For patients with stage IA2 cervical cancer with positive margins and stage IB1 with tumor measuring $\leq 2 \mathrm{~cm}$ :

- 1a: Primary treatment is surgery (1a).

- 1e, d: Surgical treatment (1) with conization (e) with

IIB: With parametrial invasion but not up to the pelvic wall 
Figure 5: Fertility-Sparing Options for Stages IA1, IA2, IB1, and select IB2 Based on Cone Biopsy Results.

pelvic lymphadenectomy with or without para-aortic lymphadenectomy (d). Sentinel LN mapping can be considered.

- or $1 b, d$ : An alternative is surgical treatment (1) with reconization (b) with pelvic lymphadenectomy. Sentinel LN mapping can also be considered.

- 2: The second step depends on surgical findings.

For patients with stage IB1 with a tumor measuring $>2 \mathrm{~cm}$ and select cases of stage IB $2 \leq 2 \mathrm{~cm}$ :

- 1a: Primary treatment is surgery (1a).

- 1e, d: Surgical treatment (1) with radical trachelectomy (e) with pelvic lymphadenectomy with or without paraaortic lymphadenectomy (d). Sentinel LN mapping can be considered.

- 2: The second step depends on surgical findings. This does not apply to patients with small neuroendocrine or gastric type adenocarcinoma of adenoma malignum.

Sector 2: Non-Fertility Sparing Options for Stages IA1, IA2, IB1, IB2, IB3, IIA1 and IIA2 Based on Cone Biopsy Results (Figure 6)

For patients with an inoperable stage IA1 cervical cancer with negative margins and negative LVSI:

- 1 : The first step is observation (1).
- 2: The second step is surveillance.

For patients with an operable stage IA1 cervical cancer with negative margins and negative LVSI:

- 1a: Primary treatment is surgery (1a).

- 1cA: Surgical treatment (1) with simple hysterectomy (cA).

- 2: The second step is surveillance.

For patients with stage IA1 cervical cancer with margins positive for dysplasia or carcinoma but negative LVSI:

- 1a: Primary treatment is surgery (1a).

- 1b: Surgical treatment (1) with re-conization (b) with pathological re-evaluation.

- or $1 \mathrm{cA}, \mathrm{d}$ : An alternative is surgical treatment (1) with simple hysterectomy (cA) or modified RH with pelvic lymphadenectomy (d). Sentinel LN mapping can also be considered.

- 2: The second step depends on surgical findings.

For patients with stage IA1 cervical cancer with positive LVSI and Stage IA2:

- 1a: Primary treatment is surgery (1a).

- $1 \mathrm{cB}, \mathrm{d}$ : Surgical treatment (1) with modified RH (cB) 


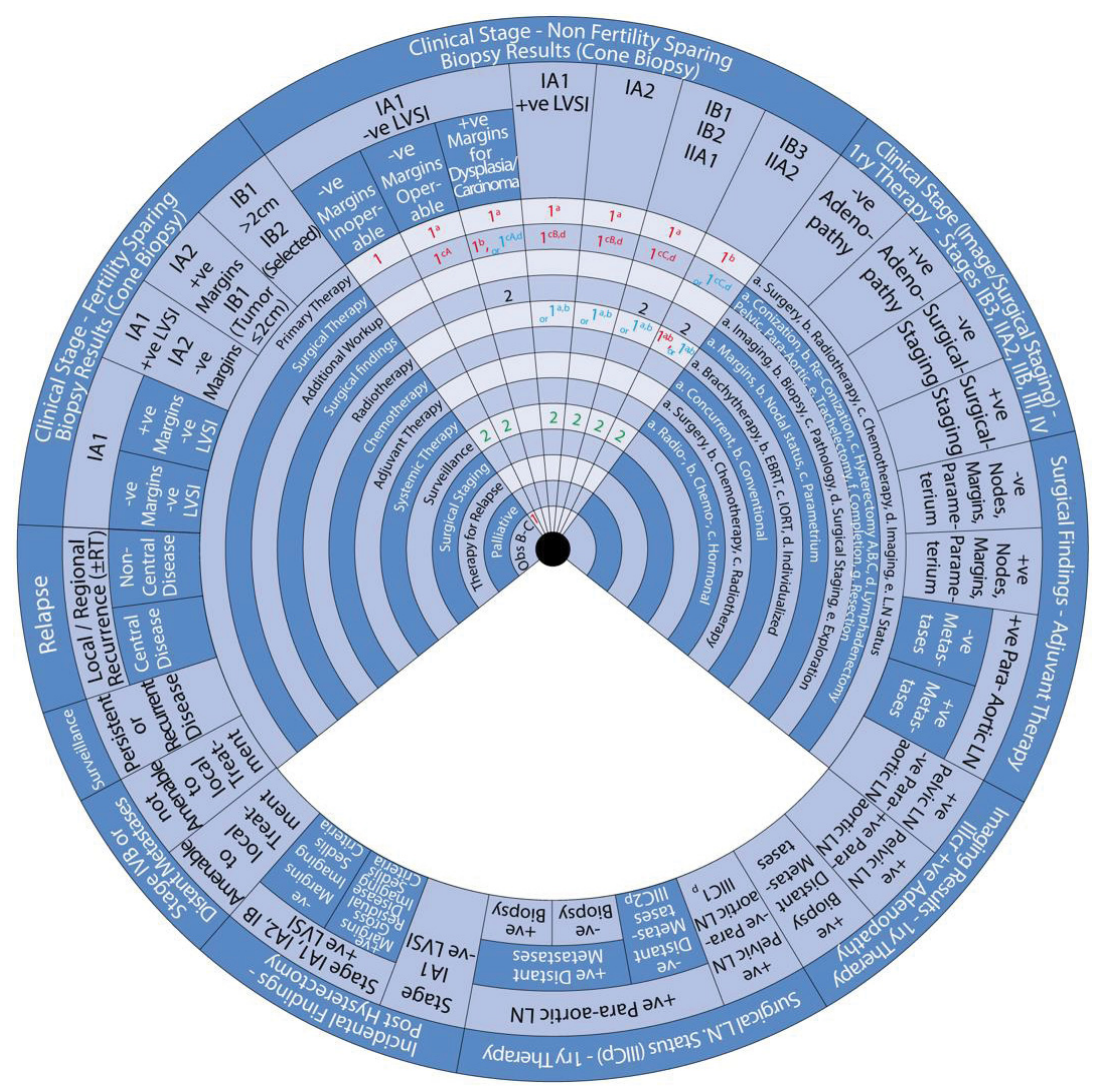

Figure 6: Non-Fertility Sparing Options for Stages IA1, IA2, IB1, IB2, IB3, IIA1 and IIA2 Based on Cone Biopsy Results.

with pelvic lymphadenectomy (d). Sentinel LN mapping can also be considered.

- or 1a, b: An alternative is a radiotherapy (1) with EBRT (b) and brachytherapy (a).

- 2: The second step is surveillance.

For patients with cervical cancer stages IB1, IB2, and IIA1:

- 1a: Primary treatment is surgery (1a).

- $1 \mathrm{cC}$, d: Surgical treatment (1) with RH (cB) and pelvic lymphadenectomy (d) with or without para-aortic lymphadenectomy. Sentinel LN mapping can also be considered.

- or 1a, b: An alternative is a radiotherapy (1) with EBRT (b) and brachytherapy (a) with or without concurrent chemotherapy.

- 2: The second step depends on surgical findings.

- 2: The next step is surveillance.

For patients with cervical cancer stages IB3 and IIA2:

- 1b: Primary treatment is radiotherapy (1b).

- 1a, b: Radiotherapy (1) with EBRT (b) and brachytherapy (a) with concurrent chemotherapy.

- or $1 \mathrm{cC}, \mathrm{d}$ : An alternative is a surgical treatment (1) with $\mathrm{RH}(\mathrm{cC})$ and pelvic lymphadenectomy (d) with or without para-aortic lymphadenectomy.

- or 1a, b: An alternative is a radiotherapy (1) with EBRT (b) and brachytherapy (a) with or without concurrent chemotherapy and adjuvant hysterectomy.

- 2: The second step depends on surgical findings.

- 2: The next step is surveillance.

Sector 3: Primary Therapy for Stages IB3, IIA2, IIB, III, and IV Based on Imaging or Surgical Staging (Figure 7)

For patients with cervical cancer stages IB3, IIA2, IIB, III, and IV and negative adenopathy:

- 1b: Primary treatment is radiotherapy (1b).

- 1a, b: Radiotherapy (1) with EBRT (b) and brachytherapy (a) with concurrent chemotherapy.

For patients with cervical cancer stages IB3, IIA2, IIB, III, and IV and positive adenopathy:

- 1a: Primary treatment is additional workup with imaging (1a). Further steps depend on the results.

For patients with cervical cancer stages IB3, IIA2, IIB, III, and IV and negative surgical staging:

- 1b: Primary treatment is radiotherapy (1b). 


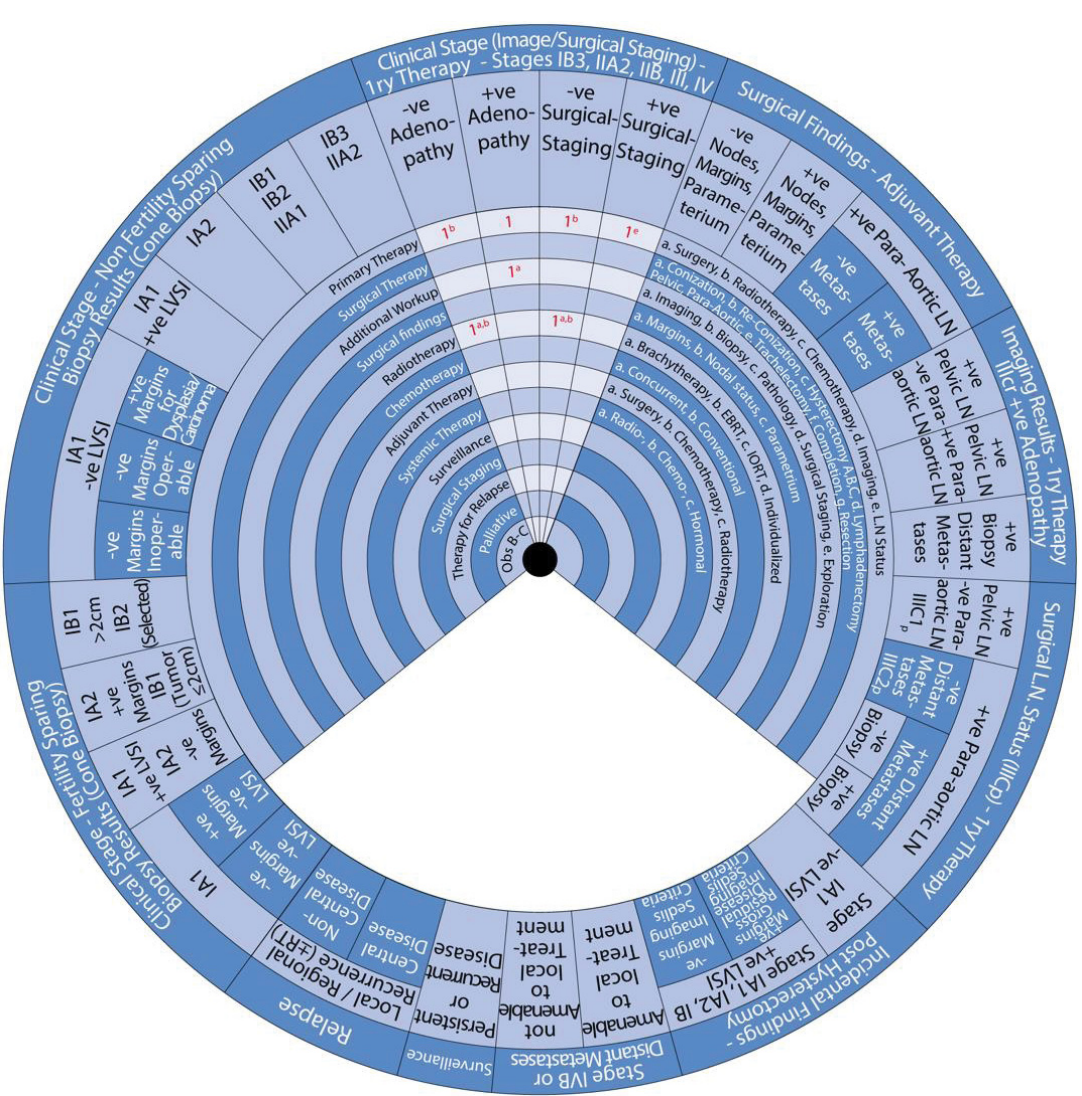

Figure 7: Primary Therapy for Stages IB3, IIA2, IIB, III, and IV Based on Imaging or Surgical Staging.

- 1a, b: Radiotherapy (1) with pelvic EBRT (b) and brachytherapy (a) with concurrent chemotherapy.

For patients with cervical cancer stages IB3, IIA2, IIB, III, and IV and positive surgical staging:

- 1e: Primary treatment depends on LN status after surgical staging (1e).

Sector 4: Adjuvant Therapy Based on Surgical Findings (Figure 8)

For patients with cervical cancer and negative nodes, margins, and parametrium, the adjuvant therapy is:

- 1 : The first step is observation if there are no risk factors (1).

- 1b: Radiotherapy (1) with EBRT (b) with or without concurrent chemotherapy if risk factors are present. According to the Sedlis criteria, those risk factors include tumor size, stromal invasion, and LVSI.

- 2: The next step is surveillance.

For patients with cervical cancer and positive pelvic nodes, margins, or parametrium, the adjuvant therapy involves:

- 1a: Primary treatment is additional workup with imaging (1a).
- 1a, b: Radiotherapy (1) with EBRT (b) and concurrent chemotherapy with or without vaginal brachytherapy (a).

- 2: The next step is surveillance.

For patients with cervical cancer and positive para-aortic nodes and negative distant metastasis, the adjuvant therapy involves:

- 1a: Primary treatment is additional workup with imaging (1a).

- 1a, b: Radiotherapy (1) with EBRT (b) and concurrent chemotherapy with or without vaginal brachytherapy (a).

For patients with cervical cancer and positive para-aortic nodes and positive distant metastasis, the adjuvant therapy involves:

- 1a: Primary treatment is additional workup with imaging (1a) and multiple biopsies (b).

- 1a, b: Radiotherapy (1) with extended-field EBRT (b) and concurrent chemotherapy with or without brachytherapy (a).

- or $1 \pm \mathrm{a}:$ An alternative is systemic therapy (1) with or without $( \pm)$ radiotherapy with EBRT (a). 
Figure 8: Adjuvant Therapy Based on Surgical Findings.

Sector 5: Primary Therapy Based on Imaging Results Demonstrating Positive Adenopathy (Figure 9)

For patients with cervical cancer and positive pelvic LN but negative para-aortic LN based on imaging:

- 1b: Primary treatment is radiotherapy (1b).

- 1a, b: Radiotherapy (1) with pelvic EBRT (b) and brachytherapy (a) with concurrent chemotherapy with or without para-aortic LN EBRT.

- or 1: An alternative is surgical staging (1) of para-aortic LN.

- 2: The second step is surveillance.

For patients with cervical cancer and positive pelvic LN and para-aortic LN based on imaging:

- 1b: Primary treatment is radiotherapy (1b).

- 1a, b: Radiotherapy (1) with extended-field EBRT (b) and brachytherapy (a) with concurrent chemotherapy.

- 2: The second step is surveillance.

For patients with cervical cancer and positive adenopathy and distant metastases based on imaging:

- 1a: Primary treatment is systemic therapy (1) with radiotherapy (a).
- $\pm 1 \mathrm{~d}$ : With or without $( \pm)$ individualized radiotherapy (1d).

Sector 6: Primary Therapy Based on Surgical Lymph Node Status (Stage IIICp) (Figure 10)

For patients with stage IIIC1p cervical cancer with positive pelvic LN but negative para-aortic LN based on surgical LN status:

- 1b: Primary treatment is radiotherapy (1b).

- 1a, b: Radiotherapy (1) with pelvic EBRT (b) and brachytherapy (a) with concurrent chemotherapy.

For patients with stage IIIC2p cervical cancer with positive para-aortic LN and negative distant metastasis based on surgical LN status:

- 1b: Primary treatment is radiotherapy (1b).

- 1a, b: Radiotherapy (1) with extended-field EBRT (b) and brachytherapy (a) with concurrent chemotherapy.

For patients with cervical cancer with positive para-aortic LN and positive distant metastasis on imaging but not by biopsy:

- 1b: Primary treatment is radiotherapy (1b).

- 1a, b: Radiotherapy (1) with extended-field EBRT (b) and brachytherapy (a) with concurrent chemotherapy. 


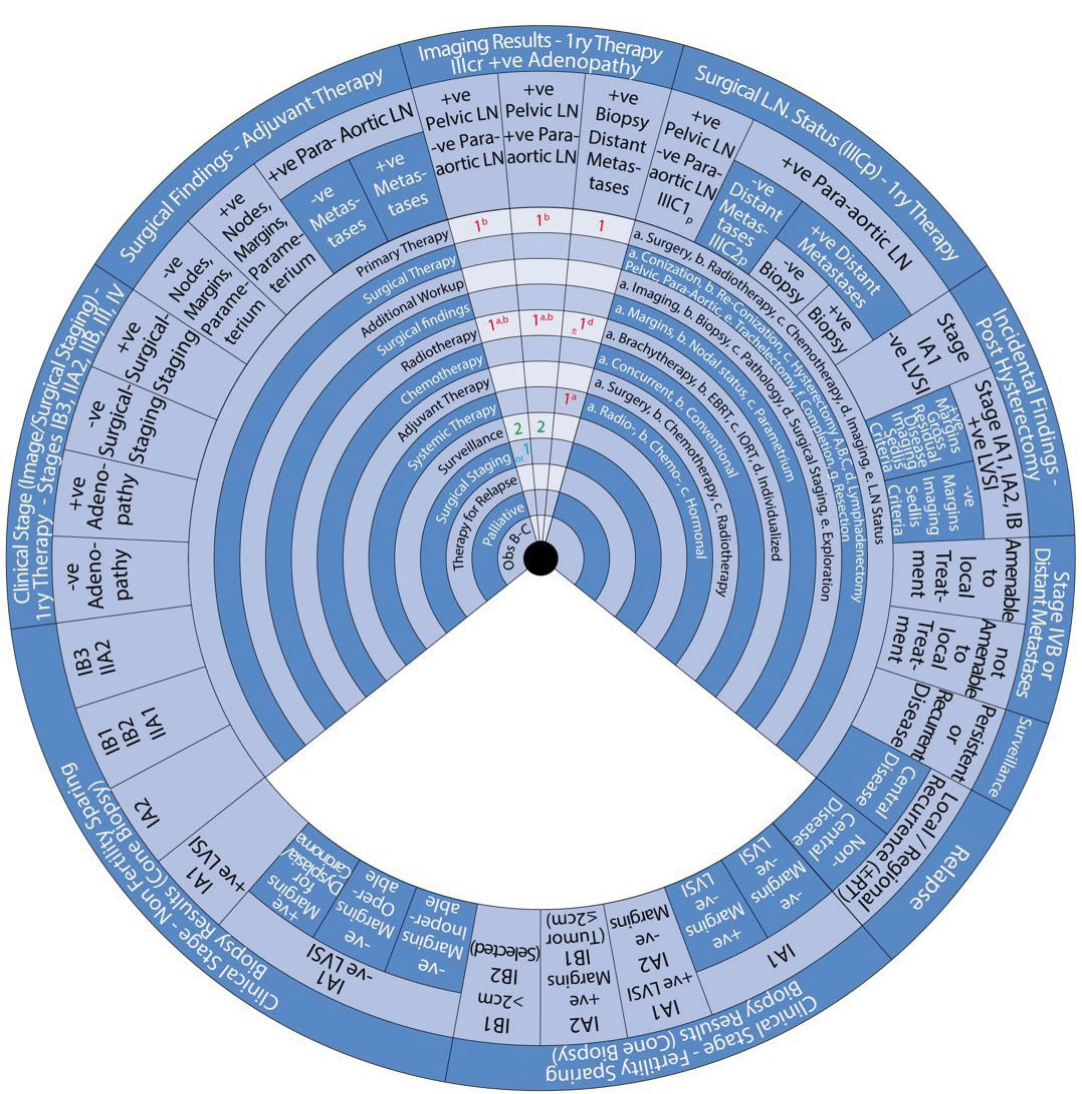

Figure 9: Primary Therapy Based on Imaging Results Demonstrating Positive Adenopathy.

For patients with Stage IIIC2p cervical cancer with positive para-aortic LN and positive distant metastasis on imaging and biopsy:

- 1: Primary treatment is systemic therapy (1).

- $\pm 1 \mathrm{~d}$ : With or without $( \pm)$ individualized radiotherapy (1d).

Sector 7: Incidental Surgical Finding of Cervical Cancer Post-Hysterectomy (Figure 11)

For patients with an incidental finding of Stage IA1 cervical cancer with negative LVSI post-hysterectomy:

- 1: Management involves surveillance (1).

7.2 Stages IA1, IA2, and IB with Positive LVSI, Negative Margins and Negative Imaging.

$1^{\text {b,a }} \quad$ Radiotherapy Pelvic EBRT+ Brachytherapy \pm Conc. Chemotherapy

or 2 Or Surgical TherapyParametrectomy, Vaginectomy + Pelvic Lymphadenectomy \pm Para-aortic Lymphadenectomy

$2^{\text {b,a }}$ Radiotherapy Pelvic EBRT + Brachytherapy \pm Conc. Chemotherapy

3 Observation if Negative $L N$ and No Residual disease

$3^{\text {b }}$ Radiotherapy Pelvic/Para-aortic EBRT \pm Brachytherapy + Conc. Chemotherapy (if Positive $L N$ or Residual disease)

For patients with an incidental finding of stage IA1, IA2, or IB cervical cancer with positive LVSI but negative margins and negative imaging post-hysterectomy:

- 1b: Primary treatment is radiotherapy (1b).
- 1a, b: Radiotherapy (1) with extended-field EBRT (b) and brachytherapy (a) with or without concurrent chemotherapy.

- or 2: An alternative is a surgical treatment (2) with parametrectomy, vaginectomy, and pelvic lymphadenectomy with or without para-aortic lymphadenectomy.

- or $2 \mathrm{~b}, \mathrm{a}$ : Another alternative is radiotherapy (2) with pelvic EBRT (b) and brachytherapy (a) with or without concurrent chemotherapy.

- 3: The next step is observation (3) if negative LN and no residual disease.

- 3b: The next step is radiotherapy (3) with pelvic/paraaortic EBRT (b) with or without brachytherapy and concurrent chemotherapy if positive LN or residual disease.

For patients with an incidental finding of Stage IA1, IA2, or IB cervical cancer with positive LVSI, margins, and imaging post-hysterectomy:

- 1a: Primary treatment is additional workup with imaging (1a) and further management depends on the findings.

Sector 8: Stage IVB or Distant Metastases (Figure 12) 


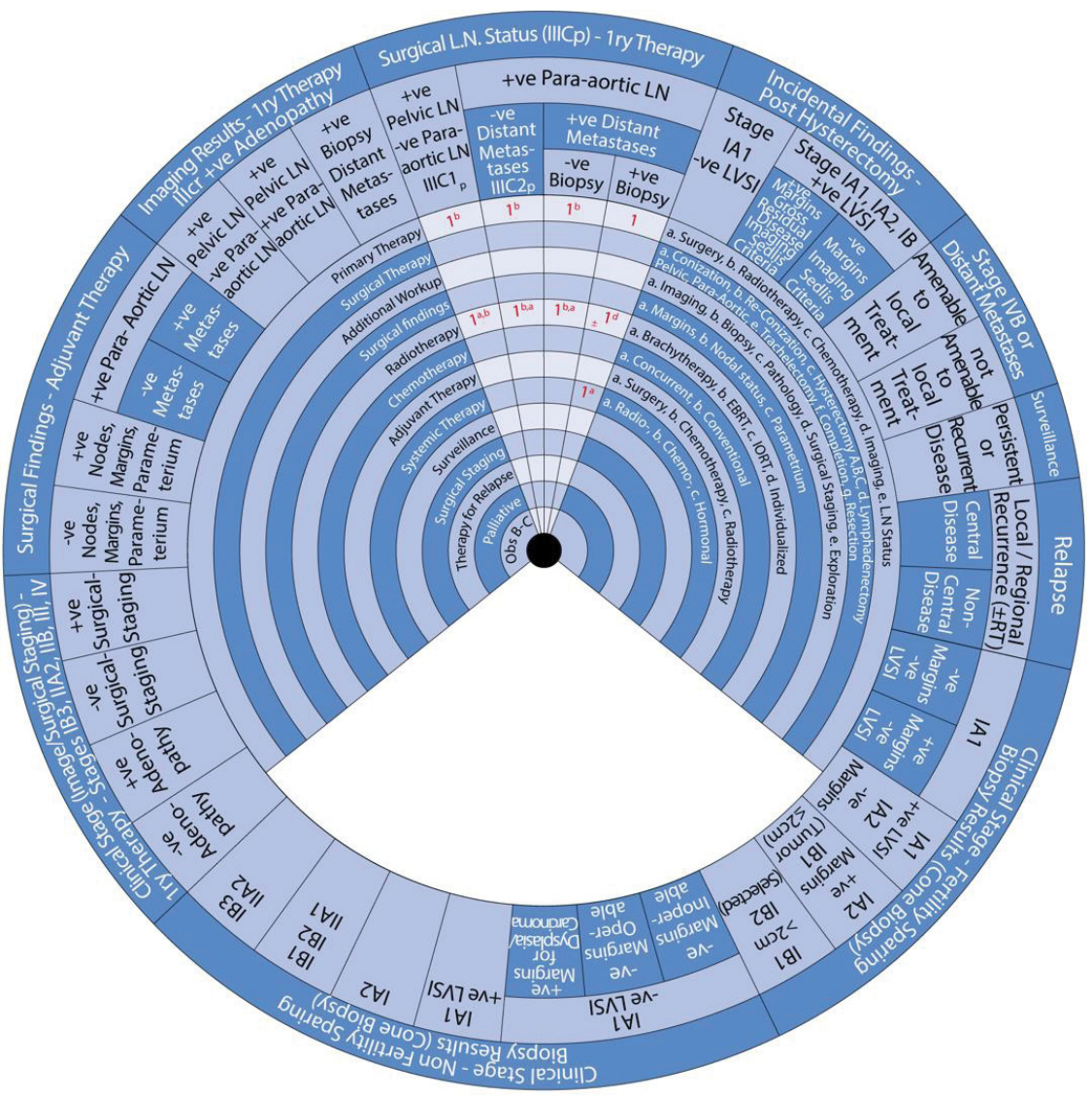

Figure 10: Primary Therapy Based on Surgical Lymph Node Status (Stage IIICp).

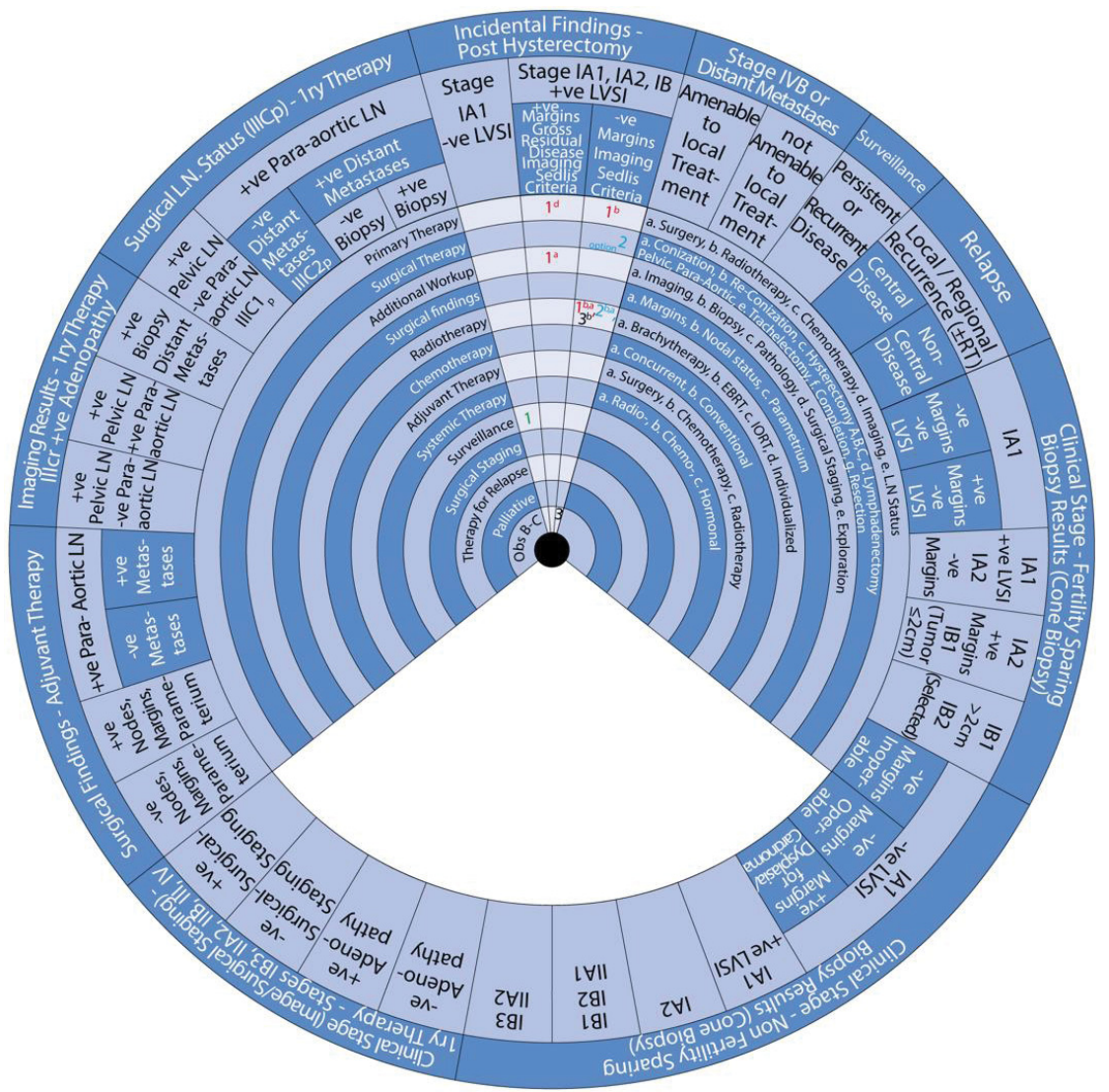

Figure 11: Incidental Surgical Finding of Cervical Cancer Post-Hysterectomy

Citation: Ismail MS, Ismail F, Ismail J, AlKhalifa M, Ismael R, et al. (2022) Development of a management guide wheel for cervical cancer. Ann Cytol Pathol 7(1): 014-028. DOI: https://dx.doi.org/10.17352/acp.000025 
For patients with stage IVB cervical cancer or distant metastases amenable to local treatment:

- 1g: Primary treatment is surgical resection (1g).

- \pm 1 b: With or without $( \pm)$ radiotherapy (1) with individualized EBRT (b).

- 1: An alternative is a surgical treatment (1) with local ablation.

- $\pm 1 \mathrm{~b}$ : With or without $( \pm$ ) radiotherapy (1) with individualized EBRT (b).

- or 1b: Or radiotherapy (1) with individualized EBRT (b) alone.

- \pm 1 : With or without $( \pm)$ systemic therapy (1).

- \#2: Consider (\#) adjuvant therapy (2) with systemic therapy.

- 3: The next step is surveillance (3).

For patients with stage IVB cervical cancer or distant metastases not amenable to local treatment:

- 1 : Primary treatment is systemic therapy (1).

- or 1: An alternative is supportive care (1).

Sector 9: Surveillance of Persistent or Recurrent Disease (Figure 13)
Surveillance of persistent or recurrent disease involves:

- 1a, e: Primary treatment is additional workup (1) with imaging (a) and surgical exploration (e).

- 1 : This is followed by therapy for relapse (1).

\section{Sector 10: Therapy for Relapse (Figure 14)}

For patients with local/regional recurrence of cervical cancer with no prior RT:

- \#1g: Consider (\#) surgical treatment (1) with resection (g).

- 1b, a: An alternative is radiotherapy (1) with individualized EBRT (b) with or without brachytherapy (a).

- \pm 1 : With or without ( \pm ) systemic therapy (1).

For patients with central-local/regional recurrence of cervical cancer with prior RT:

- 1f, g: Primary therapy is surgical treatment (1) with pelvic exenteration (f, $g$ ).

- \pm 1 c: With or without ( \pm ) radiotherapy (1) with IORT (c).

- $\pm 1 \mathrm{cC}$ : An alternative is a surgical treatment (1) with $\mathrm{RH}$ (cC)

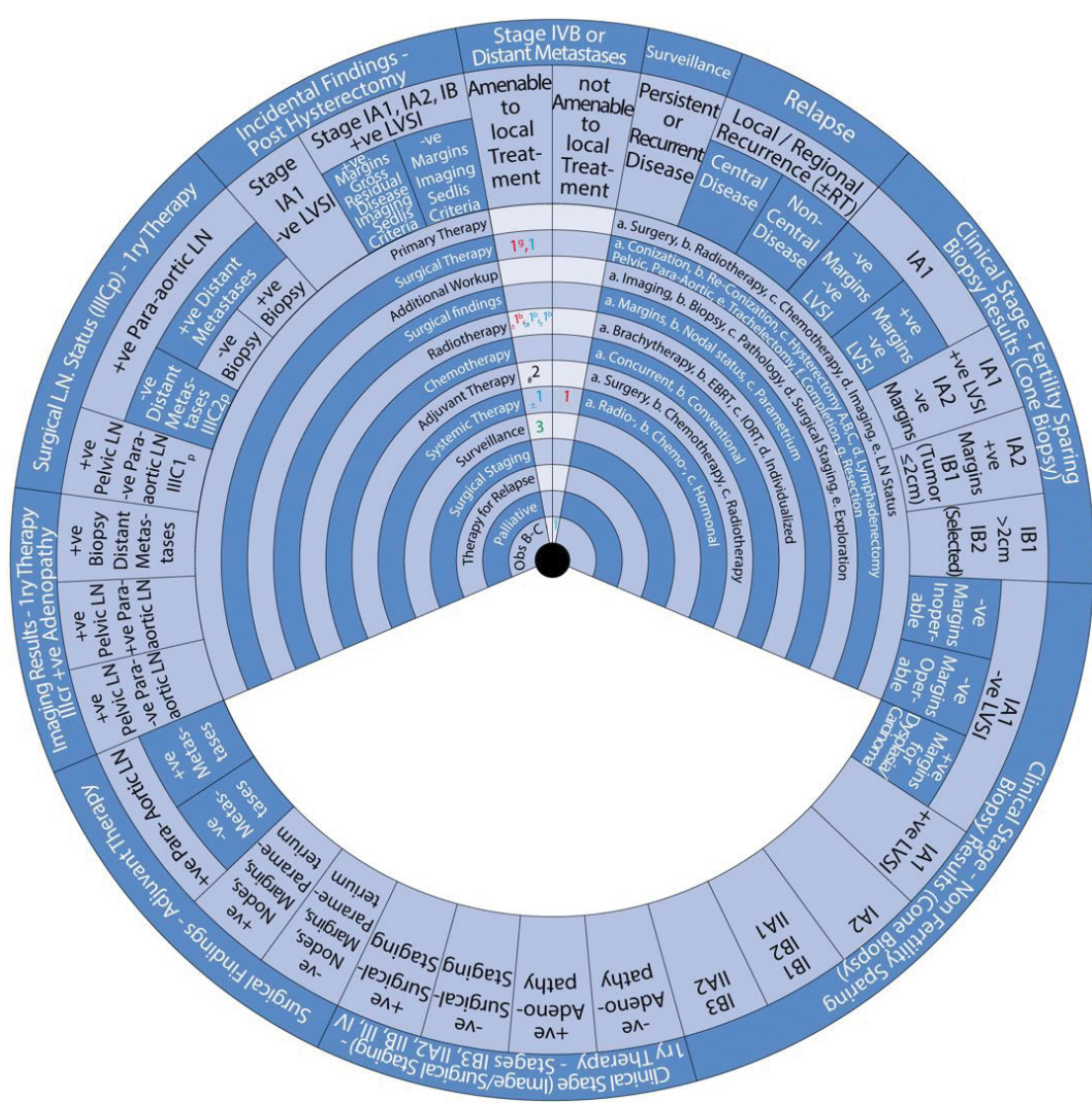




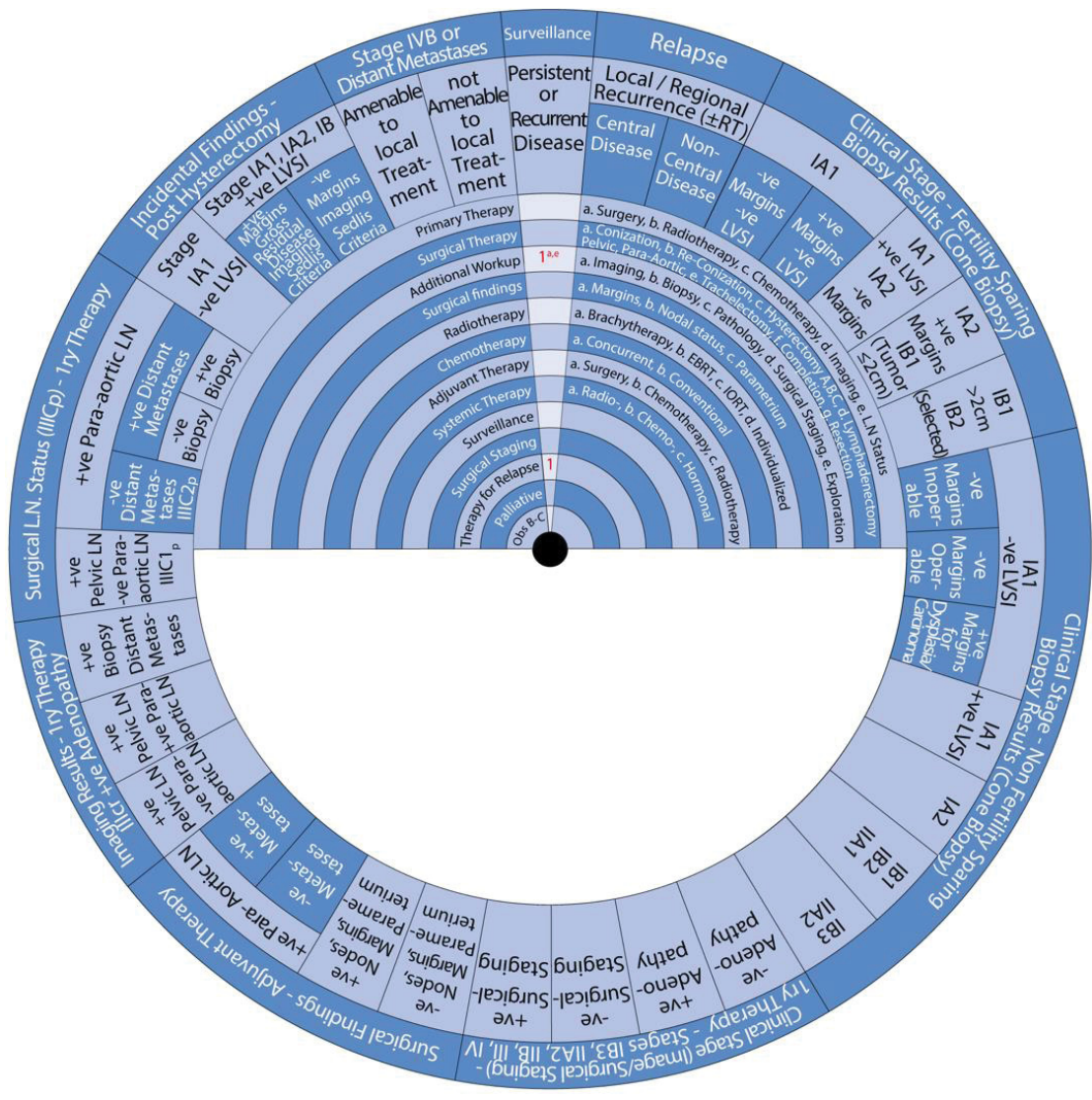

Figure 13: Surveillance of Persistent or Recurrent Disease.

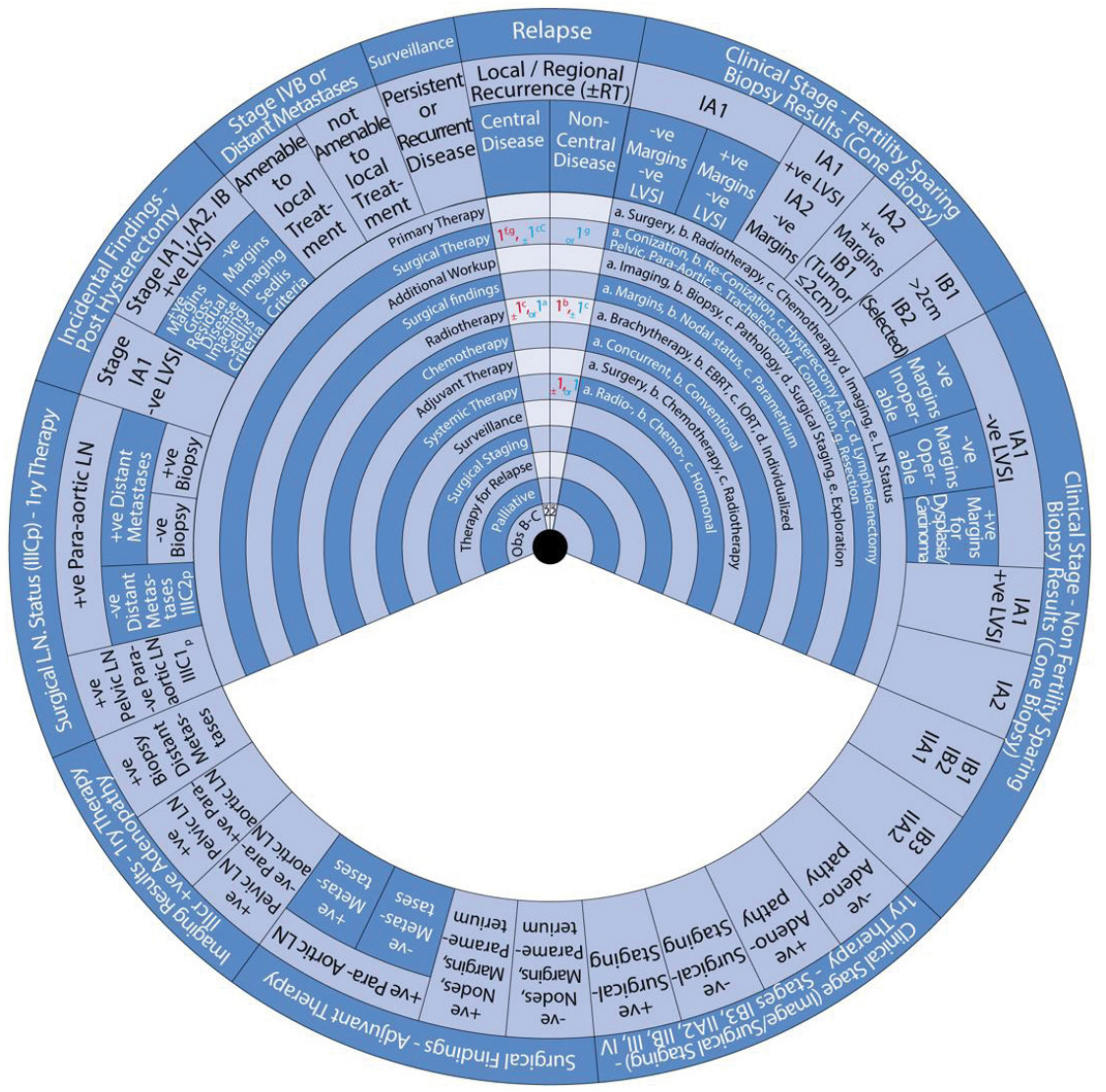

Figure 14: Therapy for Relapse.

Citation: Ismail MS, Ismail F, Ismail J, AlKhalifa M, Ismael R, et al. (2022) Development of a management guide wheel for cervical cancer. Ann Cytol Pathol 7(1): 014-028. DOI: https://dx.doi.org/10.17352/acp.000025 
- or 1a: Or radiotherapy (1) with brachytherapy (a).

- or 2: Or supportive care.

For patients with non-central local/regional recurrence of cervical cancer and prior RT:

1b: Primary therapy is surgical treatment (1) with pelvic exenteration (f, $g$ ).

- \pm 1 : With or without $( \pm)$ systemic therapy (1).

- or 1g: An alternative is a surgical therapy (1) with resection $(\mathrm{g})$.

- \pm 1 c: With or without $( \pm)$ alternative is radiotherapy (1) with IORT (c).

- or 1: Another alternative is systemic therapy (1).

- or 2: Or supportive care.

For patients with re-recurrence of cervical cancer:

- $\quad$ : Primary therapy is systemic therapy (1).

- or 1: An alternative is supportive care (1).

\section{Discussion}

A good staging system allows comparing outcomes of patients with similar characteristics within one center or across multiple centers [6]. Most guidelines including ASCO, BGCS, and NCCN use the FIGO 2018 classification for cervical cancer $[1,4,6,7,9,10,17]$. Before 2018, FIGO staged cervical cancer mainly based on clinical findings; however, in the recently updated staging system, pathological and imaging findings are taken into consideration $[1,6,7]$. Guidelines updated before 2018, such as ESGO and ESMO, rely on the Tumors, Nodes, and Metastases (TNM) staging due to this reason $[5,13]$.

Patients with CC must be assessed with a thorough history and examination in addition to laboratory investigations and imaging studies $[4,9,10,17,18]$. Laboratory investigations include a complete blood count, liver and renal function tests $[4,10,18]$. Imaging includes chest $x$-ray, computed tomography (CT), magnetic resonance imaging (MRI) or positron emission tomography/CT (PET/CT) $[4,6,9,10,17-19]$. MRI is superior to CT in detecting nodal disease but PET/CT is most sensitive [10,19-21]. Pre-operative PET/CT can help detect up to $54.8 \%$ of distant metastasis in CC [3].

Surgery is an option for early-stage CC. One option is conization and it aims to achieve negative margins $[10,18,20,21]$ Another option is hysterectomy, and there are three options: simple, modified radical, and radical $[4,7]$. Treating earlystage CC with RH and pelvic LND does not preserve fertility [17]. Fertility-sparing treatment is reserved for women with early-stage CC and good prognostic factors with no nodal disease $[13,22]$. It is not recommended for women with stage IB2 or IIA1 [10,18-20]. LVSI must be ruled out and the tumor has to measure $2 \mathrm{~cm}$ or less $[10,18]$. The presence of LVSI does not affect the staging of CC but it does affect treatment and the subsequent outcome [10]. Radical trachelectomy entails the resection of the cervix, part of the upper vagina, and the proximal parametria. It is ideally performed in the absence of LVSI, negative margins, and absent nodal disease [22]. Patients need to be counseled on the available treatment modalities and alternatives suitable for their individual case and their risks and benefits [10]. Neoadjuvant chemotherapy can help reduce the size of the tumor prior to surgery and can convert a nonfertility-sparing procedure into a more conservative approach $[9,10,18,21]$.

Sentinel lymph node (SLN) biopsy involves removing the first-draining LNs. SLN biopsy has a high sensitivity in detecting metastasis in tumors measuring $2 \mathrm{~cm}$ or less. It can be more reliable than LND to determine the nodal status [10]. Adjuvant therapy after surgery is indicated for patients with microscopic margins, nodal disease, and larger tumors $[9,10]$. It aims to reduce the risk of recurrence and increase the chance of cure [10]. Sedlis criteria can help determine those advised to receive adjuvant therapy as those with high risks including large tumor size, deep stromal invasion, and the presence of LVSI $[4,5,7,10,13]$.

A total dose of radiotherapy administered should be 4550 Gy $[4,10,18,20,21]$. Brachytherapy is a key component of RT, especially in patients with Stage IB1 or higher $[2,19,20]$. Treating women with advanced CC involves RT, including EBRT and BT, and platinum-based chemotherapy $[10,20]$. Recommended chemotherapy regimens usually include cisplatin with or without paclitaxel with or without bevacizumab $[4,5,9,10,17,21]$. Adding bevacizumab to the chemotherapy regimen is associated with improved overall survival, with more patients with a complete response without significantly reducing the quality of life [23].

Recurrence can either occur locally or distant and even both [7]. The most common site of recurrence is the pelvis [7,24]. The most common sites for distant metastasis include the lung, para-aortic LNs, and the abdominal cavity [8]. Treatment for recurrence depends on the primary therapy and the location of recurrence $[9,10,14,18]$. Unlike distant metastases, pelvic recurrences can be potentially cured with pelvic exenteration or radiation $[5,8-10,19,21,24]$. More than $90 \%$ of patients with distant metastasis will die within 5 years. Early-stage CC tends to metastasize locoregionally but can still metastasize to distant sites. Predictors of recurrence include tumor diameter, depth of stromal invasion, and the presence or absence of LVSI. If there is a high risk of recurrence after surgery, then post-operative adjuvant EBRT of the pelvis with or without chemotherapy with or without BT [8].

The aim of surveillance is to detect recurrent disease early when it can be effectively managed $[8,13,14]$. In a study by Gee et al., $13.7 \%$ of patients with locally advanced CC had "unsuspected distant metastases" [3]. The majority of recurrence occurs within 2-3 years after completing treatment and up to $95 \%$ of patients are symptomatic on presentation $[10,14,18,24]$. Follow-up is recommended every $3-4$ months for the first two years after completing treatment and every 6-12 months for three more years and then annually or as deemed 
appropriate based on the patient's individual risk of recurrence $[4,5,9,10,13,14,19-21]$.

Managing patients with CC requires a multi-disciplinary team, which consists of a gynecologist, gynecologic oncologist, histopathologist, and radiologist, among others [10,13]. It is important to consider that not all patients have access to such services, especially in isolated settings and developing countries. We designed the guide wheel to reflect the recommendations for a highly resourced setting. This wheel is intended to facilitate the referral to established recommendations on managing patients with CC. It is not meant, in any way, to replace algorithms set by the guidelines mentioned. Although this guide wheel was designed during the COVID-19 pandemic, it does not offer any recommendations on changes in practice during this time.

\section{References}

1. Manganaro L, Lakhman Y, Bharwani N, Gui B, Gigli S, et al. (2021) Staging recurrence and follow-up of uterine cervical cancer using MRI: Updated guidelines of the European Society of Urogenital Radiology after revised FIGO staging. Eur Radiol 31: 7802-7816. Link: https://bit.ly/3gyRDcx

2. LaVigne AW, Triedman SA, Randall TC, Trimble EL, Viswanathan AN (2017) Cervical cancer in low and middle income countries: Addressing barriers to radiotherapy delivery. Gynecol Oncol Rep 22: 16-20. Link: https://bit.ly/3uuGW2V

3. Gee MS, Atri M, Bandos Al, Mannel RS, Gold MA, et al. (2018) Identification of Distant Metastatic Disease in Uterine Cervical and Endometrial Cancers with FDG PET/CT: Analysis from the ACRIN 6671/GOG 0233 Multicenter Trial. Radiology 287: 176-184. Link: https://bit.ly/3HCBf6K

4. Abu-Rustum NR, Yashar CM, Bradley K, Brooks R, Campos SM, et al. (2021) Cervical Cancer, Version 1. NCCN; Pennsylvania: Link: https://bit.ly/34JrR2k

5. Marth C, Landoni F, Mahner S, McCormack M, Gonzalez-Marin A, et al. (2017) Cervical cancer: ESMO Clinical practice guidelines for diagnosis, treatment and follow-up. Ann Oncol 28: iv72-iv83. Link: https://bit.ly/3owTT8F

6. Bhatla N, Berek JS, Fredes MC, Denny LA, Grenman S, et al. (2019) Revised FIGO staging for carcinoma of the cervix uteri. Int J Gynaecol Obstet 145: 129 135. Link: https://bit.ly/3GtrTc0

7. Bhatla, N, Aoki D, Sharma DN, Sankaranarayanan R (2018) Cancer of the cervix uteri. Int J Gynaecol Obstet 143: 22-36. Link: https://bit.ly/3uyKLUw

8. Elit L, Fyles AW, Devries MC, Oliver TK, Fung-Kee-Fung M, Gynecology Cancer Disease Site Group (2009) Follow-up for women after treatment for cervical cancer: a systematic review. Gynecol Oncol 114: 528-535. Link: https://bit.ly/3soOqBL

9. Chuang LT, Temin S, Camacho R, Dueñas-Gonzalez A, Feldman S, et al. (2016) Management and Care of Women with Invasive Cervical Cancer: American Society of Clinical Oncology Resource-Stratified Clinical Practice Guideline. J Glob Oncol 2: 311-340. Link: https://bit.ly/3BjDE41
10. Reed N, Balega J, Barwick T, Buckley L, Burton K, et al. (2021) British Gynaecological Cancer Society (BGCS): Cervical Cancer Guidelines: Recommendations for practice. Eur J Obstet Gynecol Reprod Biol 256: 433465. Link: https://bit.ly/3B3y0m8

11. Funston, G, O'Flynn H, Ryan NAJ, Hamilton W, Crosbie EJ (2018) Recognizing gynecological cancer in primary care: risk factors, red flags, and referrals. Adv Ther 35: 577-589. Link: https://bit.ly/3LhNs2H

12. Ismail MS, Hsu S, AlKhalifa MA, Fouad M, Codabux F, et al. (2020) Evaluation of different Guidelines for cervical cancer screening and management of abnormal cervical cytology. Ann Cytol Pathol 5: 001-012. Link: https://bit.ly/3osXsMU

13. Cibula D, Pötter R, Planchamp F, Avall-Lundqvist E, Fischerova D, et al. (2018) The European Society of Gynaecological Oncology/European Society for Radiotherapy and Oncology/European Society of Pathology guidelines for the management of patients with cervical cancer. Virchows Arch 472: 919-936. Link: https://bit.ly/3GtDXdr

14. Salani R, Khanna N, Frimer M, Bristow RE, Chen LM (2017) An update on posttreatment surveillance and diagnosis of recurrence in women with gynecologic malignancies: Society of Gynecologic Oncology (SGO) recommendations. Gynecol Oncol 146: 3-10. Link: https://bit.ly/3ovZtb8

15. Ismail MS, Ismail F, Ismail J, Rustogi A, Torsten U, et al. (2020) Development of a management guideline wheel for uterine neoplasms based on different guidelines for management of uterine neoplasms. Cancer Rep Rev 4: 1-27. Link: https://bit.ly/34qvNp9

16. Ismail MS (2020) Development of a management guideline wheel for abnorma pap smear and related cervical pathology. Ann Cytol Pathol 5: 013-034. Link: https://bit.ly/34GjqoC

17. Johnson CA, James D, Marzan A, Armaos M (2019) Cervical cancer: An overview of pathophysiology and management. Semin Oncol Nurs 35: 166174. Link: https://bit.ly/3Lfd1S9

18. Alberta Health Services. Cancer of the uterine cervix. Alberta: AHS

19. Lim MC, Lee M, Shim SH, Nam EJ, Lee JY, et al. (2017) Practice Guidelines for Management of Cervical Cancer in Korea: A Korean Society of Gynceologic Oncology Consensus Statement. J Gyencol Oncol 28: e22. Link: https://bit.ly/3JcxF3C

20. Chopra SJ, Mathew A, Maheshwari A, Bhatla N, Singh S, et al. (2018) Nationa Cancer Grid of India Consensus Guidelines on the Management of Cervical Cancer. J Glob Oncol 4: 1-15. Link: https://bit.ly/3LtUmIV

21. de Juan A, Redondo A, Rubio MJ, García Y, Cueva J, et al. (2020) SEOM clinical guidelines for cervical cancer (2019). Clin Transl Oncol 22: 270-278. Link: https://bit.ly/3gtf479

22. Bentivegna E, Gouy S, Maulard A, Chargari C, Leary A, et al. (2016) Oncologica outcomes after fertility-sparing surgery for cervical cancer: a systematic review. Lancet Oncol 17: e240-253. Link: https://bit.ly/3LhZUQk

23. Marquina G, Manzano A, Casado A (2018) Targeted agents in cervical cancer: Beyond bevacizumab. Curr Oncol Rep 20: 40. Link: https://bit.ly/3Je4YmC

24. Elit L, Reade CJ (2015) Recommendations for Follow-up Care for Gynecologic Cancer Survivors. Obstet Gynecol 126: 1207-1214. Link: https://bit.ly/3rzSNe2

Citation: Ismail MS, Ismail F, Ismail J, AIKhalifa M, Ismael R, et al. (2022) Development of a management guide wheel for cervical cancer. Ann Cytol Pathol 7(1): 014-028. DOI: https://dx.doi.org/10.17352/acp.000025 